\title{
A inhomogenous jet model for the broad band emission of radio loud AGNs
}

\author{
Thomas Vuillaume*t \\ Laboratoire d'Annecy-le-Vieux de Physique des Particules, CNRS, France \\ E-mail: thomas.vuillaume@lapp.in2p3.fr
}

\section{Gilles Henri}

IPAG, Univ. Grenoble Alpes, CNRS, France

E-mail: gilles.henriduniv-grenoble-alpes.fr

Pierre-Olivier Petrucci

IPAG, Univ. Grenoble Alpes, CNRS, France

E-mail: pierre-olivier.petruccieuniv-grenoble-alpes.fr

\begin{abstract}
We propose a coherent jet model for radio loud AGNs. This model is based on the assumption that the jet is composed of two flows: an external MHD plasma, surrounding an internal highly relativistic plasma of electron-positrons. The leptons present in the spine, responsible of most of the emission, are supposed to be continuously heated via diffuse acceleration along the jet. The model is thus naturally inhomogeneous and includes most of the expected electromagnetic processes in radio loud sources: synchrotron, self-synchrotron Compton and external Compton emission (with Klein-Nishina cross section) on external sources (accretion disk, dusty torus and broad line region), photon-photon absorption and pair creation. A key point of the model is that the jet bulk Lorentz factor is not a free parameter but is the result of the Compton rocket effect and is thus imposed by external sources, themselves constrained by observation. The particles energy distribution is computed coherently along the jet applying energy conservation between heating and cooling and flux conservation, and the emerging spectrum is computed numerically. This model is applied to the quasar 3C273 and successfully reproduces its broad band emission over 15 orders of magnitude.
\end{abstract}

35th International Cosmic Ray Conference

10-20 July, 2017

Bexco, Busan, Korea

* Speaker.

${ }^{\dagger}$ A footnote may follow. 


\section{Introduction}

Active Galactic Nuclei (AGN), and especially radio-loud ones, are famous for their powerful emission of non thermal radiation. The source of these radiations is still unclear but leptonic models seem to be favoured. In these models, leptons are accelerated to relativistic speeds in a magnetized jet and emit synchrotron and inverse Compton photons. Central components of the models such as the composition of the jet (leptonic versus baryonic), the bulk acceleration mechanism, the heating mechanism are still matter of debates. The most simple and widespread model, the so-called one-zone model, presents a single, spherical and homogeneous emission. Only a small numbers of parameters are free: the size of the zone, the particle distribution, the magnetic field value and the bulk Lorentz factor. Their advantage is to be simple and to give a good first approximation of the observed emission. However, several limitations are encountered by these models: difficulties to reproduce the lowest part of the observed spectra (in radio) or to explain in details the variability of the sources. Moreover, as their physics is rather limited, they do not a provide a global understanding of the jets.

To overcome these issues, more complex models have been proposed such as the blob-in-jet models [11, 10] or the structured jet model proposed by [7]. The idea of a multiple-components jet, namely the "two-flow" model (see section 2), was first proposed by [24]. Their original idea was to propose a double jet structure for AGN jets: a outer jet, or sheath, formed by the accretion disc, baryonic and mildly relativistic would contain an inner beam, leptonic and moving at much higher Lorentz factors. These assumption, based on theoretical reasons have been revived by recent observations [14, 4].

Subsequent works $[12,13,20,21,6,26]$ have developed the two-flow paradigm, enriched by theoretical developments and numerical developments.

Here we present a complete numerical model of a stratified jet in the two-flow paradigm. We first present the assumption and general ideas of the two-flow paradigm, then develop the numerical model to finally apply it to the quasar 3C273, successfully reproducing its broad-band emission.

\section{The two-flow paradigm}

Based on the original idea from [24], the "two-flow" has since evolved. In this paradigm, the jet is actually composed of two different flows. The external sheath is a MHD jet, or wind, fuelled by the accretion disc. Produced by the Blandford \& Payne mechanism ([3]) it is self-collimated and much alike jets found in other astrophysical objects such as young stars or neutron stars. As such, it is a baryonic mildly relativstic jet $(\beta \approx 0.5)$. The empty funnel created by this mechanism is leaves a the opportunity for another jet to develop. This inner jet made of relativistic leptons $\left(e^{-} / e^{+}\right)$ is created by $\gamma-\gamma$ interactions between soft and high-energy photons. This flow is confined by the outer jet and continuously reheated by the interaction with the MHD flow, so that it can be accelerated to highly relativistic speeds through the Compton rocket effect (see below).

The advantages of this whole construction are various:

- It solves the confinement issue of a highly relativistic flow which cannot be self-confined [5], [16]. 
- The interaction arising between the MHD sheath and the inner flow is an elegant way to accelerate particles throughout the jet via turbulence. In this case, the sheath can be seen as an energy reservoir carrying most of the power in which particles can tap energy.

- Because particles are continuously reheated along the jet, the Compton rocket effect first introduced by [15] can arise. In this process, the inverse Compton scattering of photons in a particular direction induces a momentum of the emitting particles in the opposite direction. The arguments developed by [17] showing that the jet cannot reach high bulk Lorentz factors as the particles quickly cool does not hold here as the particles have a constant source of energy.

- It can help solving the bulk Lorentz factor crisis [9] presenting a divergence between the high Lorentz factors required to explain the blazars fast variability [1] and to overcome the $\gamma$-ray opacity [2] and the ones observed at parsec scales by VLBI measurements [18, 8, 19]. The discrepancy can arise from the presence of the two flows.

- The spine/sheath structure could solve the unification problem between FR I and BL Lacs objects concerning their Lorentz factors [7].

- Recent observations indicates complex structures in jet close to the one described by the two-flow $[14,4]$.

In figure 1 below is presented a scheme of the two-flow structure.

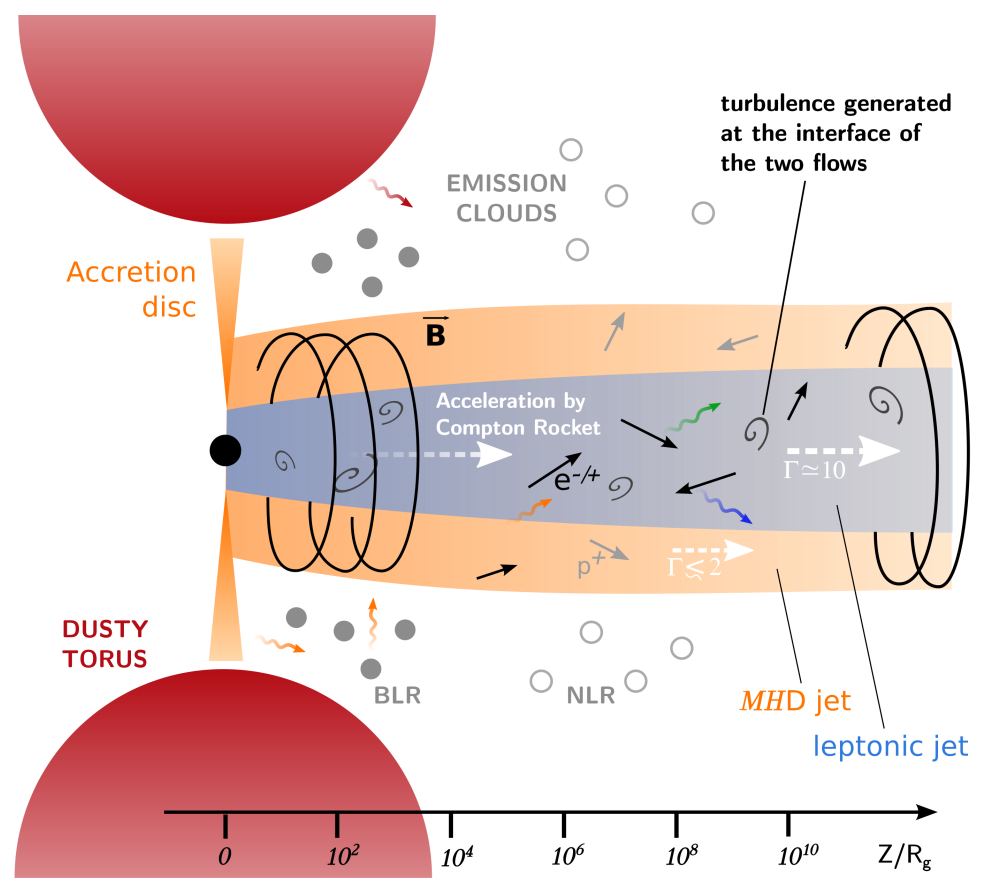

Figure 1: Schematic view of the two-flow paradigm. 


\section{Numerical modeling}

A numerical modeling of the jet in the two-flow paradigm has been proposed by [21] and developed by [6]. In this model, the jet follow a stratification along its main axis (called $z$ in figure 1). Numerically, the jet is sliced with an adaptative grid and physical conditions are computed in each slice, thus evolving along $z$. Initial conditions are given at a fixed altitude in the jet. Each slice can be represented as a one-zone model of its own emitting synchrotron radiation and inverse Compton radiation on synchrotron soft photons (synchrotron self-Compton or SSC) or on external soft photons (external Compton or EC). External sources of soft photons are also modelled. They are essential for the EC emission as well as for the induced Compton rocket force on the plasma. The opacity due to $\gamma-\gamma$ interaction is also computed coherently with these sources.

\subsection{Jet geometry}

Three ad-hoc laws model the evolution of the physical parameters in the jet.

The radius of the spine (equivalent to the inner radius of the sheath) is described by a paraboloid of minimal radius the gravitational radius $R_{g}=G M_{b h} / c^{2}$. The parabola is displaced by the constant $R_{i}$ to avoid divergence issues when $z$ tends to 0 . The jet opening is given by the index $w$. A collimated jet requires $w<1$.

$$
R(z)=R_{0}\left[\frac{z}{Z_{0}}+\left(\frac{R_{i}}{R_{0}}\right)^{1 / \omega}\right]^{\omega} \quad \text { with } \quad \omega<2
$$

The magnetic field is homogeneous in a slice and its evolution along $\mathrm{z}$ is given by:

$$
B(z)=B_{0}\left(\frac{R(z)}{R_{0}}\right)^{-\lambda} \quad \text { with } \quad 1<\lambda<2
$$

An index $\lambda=1$ would correspond to a pure toroidal magnetic field while an index $\lambda=2$ corresponds to a purely poloidal magnetic field.

In this model, the particles energy is not prescribed directly but through their heating term $Q_{a c c}$ following the equation:

$$
Q_{a c c}(z)=Q_{0}\left[\frac{z}{Z_{0}}+\left(\frac{R_{i}}{R_{0}}\right)^{1 / \omega}\right]^{-\zeta} \exp \left(-\frac{z}{Z_{c}}\right)
$$

The altitude $Z_{c}$ corresponds to the end of the energy transfer to the particles. Similarly to the jet radius expression (equation (3.1)), the constant $R_{i} / R_{0}$ avoids numerical issues when $\mathrm{Z}$ tends to 0 .

The logarithmic sampling of frequencies between $v_{\min }$ and $v_{\max }$ is done in the observer frame. It is therefore dependent of the Lorentz factor in the jet frame. This ensures that local emissivities are computed for the same sampling (the observer one) when transferred in the observer frame.

\subsection{Geometry of the external sources}

In this model, the three main sources of external photons in AGN are present: the accretion disc, the dusty torus and the broad line region (BLR). Their geometry is the same used and described in [26]. 
- The accretion disc is described by a plane disc perpendicular to the jet at $z=0$. It is sliced for numerical integration. Its emission is the one of a standard accretion disc with a radial temperature distribution: $T_{\text {disc }}(r)=\left[\frac{3 G M \dot{M}}{8 \pi \sigma} \frac{1}{r^{3}}\left(1-\sqrt{\frac{3 R_{S}}{r}}\right)\right]^{1 / 4}$ [23].

- The dusty torus is modeled as a geometrical torus outside the accretion disc. It is sliced for numerical integration and each slice is in radiative equilibrium with the radiation coming from the accretion disc, imposing a local temperature. The slices then emit at grey bodys.

- The BLR is modeled has a spherical shell of geometrically and optically thin clouds at a distance $R_{b l r}$ from the central black-hole. It is therefore located above the accretion disc and absorbs and re-emits part of its luminosity. Its emits as a grey-body at a temperature of $T=10^{5} \mathrm{~K}$ and an emissivity computed so that the luminosity corresponds to a fixed fraction of the disc luminosity.

\subsection{Particle distribution and emitting processes}

As we deal with emitting leptons here, only synchrotron and inverse Compton radiations need to be considered. The considered sources of soft photons for inverse Compton are plural: synchrotron photons give synchrotron (SSC) and external photons (from the accretion disc, the dusty torus and the BLR) give external Compton (EC). Emission is computed in the jet rest frame and then boosted by the local Doppler factor $\delta(z)=\left(\Gamma(z)\left(1-\beta(z) \mu_{i}\right)^{-1}\right.$ (with $\mu_{i}$ the cosinus of the observation angle with respect to the jet axis and $\beta$ and $\Gamma$ the jet speed and Lorentz factor) into the observer's rest frame.

In the two-flow paradigm, particles are accelerated through turbulence processes. This kind of processes are described by the Fokker-Planck equation which leads to quasi-Maxwellian distributions ([22]). We adopt the following simplified form for the particle distribution:

$$
n_{e}(\gamma, z)=n_{0}(z) \frac{\gamma^{2}}{2 \bar{\gamma}^{3}(z)} \exp (-\gamma / \bar{\gamma}(z))
$$

where $n_{0}(z)=\int n_{e}(\gamma) \mathrm{d} \gamma$.

Parameters of the distribution $\left(n_{0}(z)\right.$ and $\left.\bar{\gamma}(z)\right)$ are computed coherently from the physical conditions in the jet and the emission.

The particles characteristic energy $\bar{\gamma}(z) m_{e} c^{2}$ results from the balance between heating and radiative cooling. In our model, heating is described by the ad-hoc parameter $Q_{a c c}(z)$ (see Eq. 3.3). The particles cooling however is due to their emission in the comoving frame. Considering that most of the efficient cooling is happening in the Thomson regime, the particles characteristic energy at every step can be determined solving equation 3.5 :

$$
\frac{\partial \bar{\gamma}}{\partial t}(z, t)=\delta_{b}(z)\left[Q_{a c c}(z)-\frac{4}{3} \frac{\sigma_{T}}{m_{e} c}\left(U_{B}+U_{s y n c}(\bar{\gamma})+U_{e x t}(\bar{\gamma})\right)\left(\bar{\gamma}^{2}-1\right)\right]
$$

where $U_{B}, U_{s y n c}$ and $U_{e x t}$ are the total energy densities of the photon fields related to respectively the synchrotron, SSC and EC emissions. 
The particle density evolution is the result of pair production which is a direct consequence of the $\gamma-\gamma$ absorption in the jet.

\subsection{Evolution of the jet bulk Lorentz factor}

As explained in the section 2, the dynamic of the jet is driven by the Compton rocket process [15] in the two-flow paradigm as described here. Therefore, the bulk Lorentz factor of the jet $\Gamma_{b}(z)$ is not a free parameter of the model but as shown by [26] is imposed by the external sources. We use the method described in [26] to compute the evolution of $\Gamma_{b}(z)$ along the jet thanks to the external sources geometry described previously and to the physical conditions in the jet.

\section{Example of application: the quasar $3 \mathrm{C} 273$}

The model has been successfully applied to the quasar 3C273 giving a very good description of its broadband emission from radio to very-high energies. The resulting SED is given in figure 2.

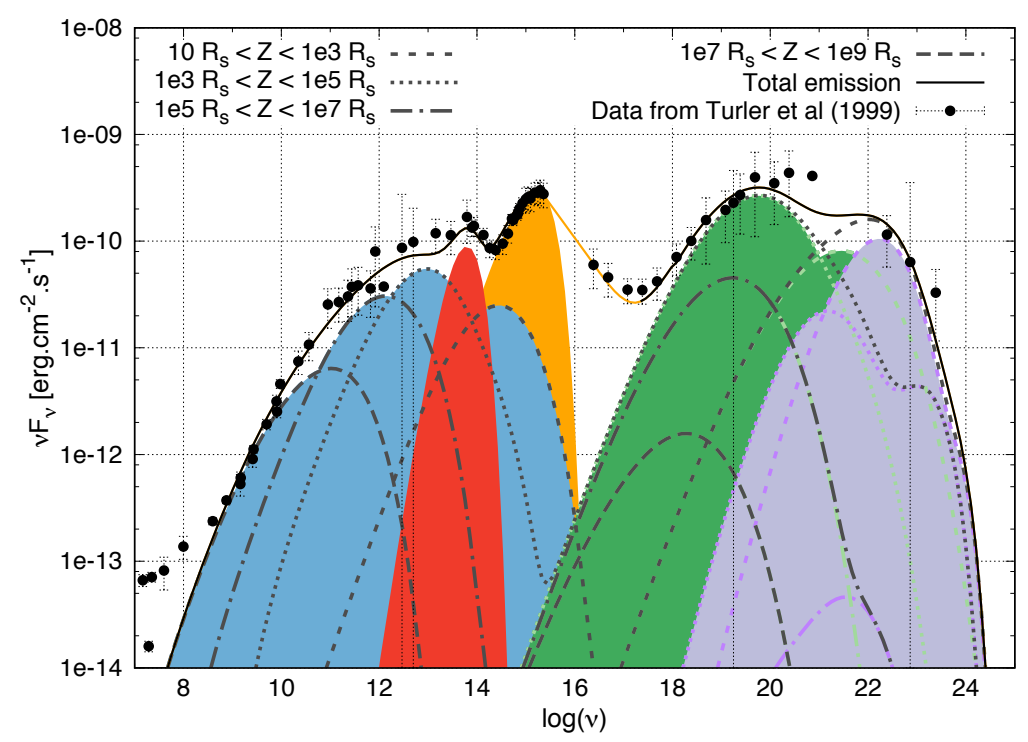

Figure 2: SED of 3C 273 - modeling compared to data from [25]. In blue the synchrotron emission, in green the SSC and in purple the external Compton. In red is the torus emission and in orange is the multicolor accretion disc completed with a power-law describing the hot corona emission between 0.02 and $200 \mathrm{keV}$. Different emission zones in the jet are represented with different dotted lines.

As one can see the different processes and different altitudes explain different parts of the observed SED and the complete jet is required to explain the whole emission. The radio emission is mainly produced at large scale and the synchrotron emission peak decrease with the altitude. The very-high energy emission however is produced by inverse Compton (SSC and EC) in central regions (below $10^{3} R_{s}$ ). 


\section{Conclusion \& Acknowledgement}

We presented a numerical model of a stratified jet in the two-flow paradigm and applied it successfully to explain the observed broadband emission of the AGN 3C273. The two-flow model has the advantages of providing a complex understanding of AGN jets while imposing strong constraints on the parameters such as the jet bulk Lorentz factor. It is moreover in agreement with recent observations showing complex structures and behaviour in jets at all scales.

This work has been partly funded by the french CNES agency.

\section{References}

[1] F Aharonian et al. An Exceptional Very High Energy Gamma-Ray Flare of PKS 2155-304. Astrophysical Journal, 664(2):L71-L74, July 2007.

[2] Mitchell C Begelman, Andrew C Fabian, and Martin J Rees. Implications of very rapid TeV variability in blazars. Monthly Notices of the Royal Astronomical Society: Letters, 384(1):L19-L23, February 2008.

[3] R D Blandford and D G Payne. Hydromagnetic flows from accretion discs and the production of radio jets. Monthly Notices of the Royal Astronomical Society, 199:883-903, June 1982.

[4] B Boccardi, T P Krichbaum, U Bach, F Mertens, E Ros, W Alef, and J A Zensus. The stratified two-sided jet of CygnusâĂL'A. Astronomy and Astrophysics, 585:A33, December 2015.

[5] S V Bogovalov. Acceleration and collimation of relativistic plasmas ejected by fast rotators. Astronomy and Astrophysics, 371(3):1155-1168, June 2001.

[6] T Boutelier, G Henri, and P-O Petrucci. An inhomogeneous jet model for the rapid variability of TeV blazars. Monthly Notices of the Royal Astronomical Society: Letters, astro-ph(1):L73-L77, July 2008.

[7] G Ghisellini, F Tavecchio, and M Chiaberge. Structured jets in TeV BL Lac objects and radiogalaxies. Astronomy and Astrophysics, 432(2):401-410, March 2005.

[8] M Giroletti, G Giovannini, L Feretti, W D Cotton, P G Edwards, L Lara, A P Marscher, J R Mattox, B G Piner, and T Venturi. Parsec-Scale Properties of Markarian 501. The Astrophysical Journal, 600(1):127-140, January 2004.

[9] G Henri and L Saugé. The Bulk Lorentz Factor Crisis of TeV Blazars: Evidence for an Inhomogeneous Pileup Energy Distribution? The Astrophysical Journal, 640(1):185-195, March 2006.

[10] O Hervet, C Boisson, and H Sol. Linking radio and gamma ray emission in Ap Librae. arXiv.org, March 2015.

[11] K Katarzynski, H Sol, and A Kus. The multifrequency emission of Mrk 501. Astronomy and Astrophysics, 367(3):809-825, March 2001.

[12] A Marcowith, G Henri, and G Pelletier. Gamma-ray emission of blazars by a relativistic electron-positron beam. Monthly Notices of the Royal Astronomical Society, 277(2):681-699, November 1995.

[13] A Marcowith, G Pelletier, and G Henri. Acceleration mechanism in Blazar jets. Astronomy and Astrophysics Supplement, 120:563, December 1996. 
[14] F Mertens and A Lobanov. Longitudinal and transverse velocity fields in parsec-scale jets. eprint arXiv:1505.06951, pages -, May 2015.

[15] S L O'dell. Radiation force on a relativistic plasma and the Eddington limit. Astrophysical Journal, 243:L147-L149, February 1981.

[16] G Pelletier. Black Hole Induced Ejections. eprint arXiv:astro-ph/0405113, May 2004.

[17] E S Phinney. Acceleration of a Relativistic Plasma by Radiation Pressure. Monthly Notices of the Royal Astronomical Society, 198:1109, March 1982.

[18] B Glenn Piner and Philip G Edwards. The Parsec-Scale Structure and Jet Motions of the TeV Blazars 1ES 1959+650, PKS 2155-304, and 1ES 2344+514. Astrophysical Journal, 600(1):115-126, January 2004.

[19] B Glenn Piner and Philip G Edwards. FIRST-EPOCH VLBA IMAGING OF 20 NEW TeV BLAZARS. Astrophysical Journal, 797(1):25, December 2014.

[20] N Renaud and G Henri. The terminal bulk Lorentz factor of relativistic electron-positron jets. Monthly Notices of the Royal Astronomical Society, 300(4):1047-1056, November 1998.

[21] L Saugé and G Henri. TeV Blazar GammaâĂŘRay Emission Produced by a Cooling Pileup Particle Energy Distribution Function. Astrophysical Journal, 616(1):136-146, November 2004.

[22] R Schlickeiser. An explanation of abrupt cutoffs in the optical-infrared spectra of non-thermal sources - A new pile-up mechanism for relativistic electron spectra. Astronomy and Astrophysics, 136:227-236, July 1984.

[23] N I Shakura and R A Sunyaev. A theory of the instability of disk accretion on to black holes and the variability of binary X-ray sources, galactic nuclei and quasars. Monthly Notices of the Royal Astronomical Society, 175:613-632, June 1976.

[24] H Sol, G Pelletier, and E Asseo. Two-flow model for extragalactic radio jets. Monthly Notices of the Royal Astronomical Society (ISSN 0035-8711), 237:411-429, March 1989.

[25] M Turler, S Paltani, T J L Courvoisier, M F Aller, H D Aller, A Blecha, P Bouchet, M Lainela, I M McHardy, E I Robson, J A Stevens, H Terasranta, M Tornikoski, M H Ulrich, E B Waltman, W Wamsteker, and M C H Wright. 30 years of multi-wavelength observations of 3C 273. arXiv.org, pages 89-101, July 1998.

[26] T Vuillaume, G Henri, and P O Petrucci. Variation of bulk Lorentz factor in AGN jets due to Compton rocket in a complex photon field. Astronomy and Astrophysics, 581:A18-, August 2015. 